\title{
Intuitionistic Fuzzy Neural Networks based on Extended Kalman Filter Training algorithm
}

\author{
Xiaoguang Zhou, Renhou Zhao, Xiumin Shang \\ Flight Simulation Training Department \\ Naval Aviation Institute \\ Hu Ludao,LiaoNing, 125001,China \\ zhanglili2117@163.com
}

Abstract-In this paper, an intuitionistic fuzzy neura network model is proposed. The network structure has five layers, and adopts Mandani's fuzzy reasoning. A new fuzzy inference system is applied in the model, which contains hesitation margin as a part. A training algorithm based on Extended Klaman Filter(EKF) is development. The EKF procedure to update parameter is introduced. The derivation of EKF based on adaptation algorithm for intuitionistic fuzzy adaptive equalizer is given. An example is given to demonstrate the intuitionistic fuzzy neural network based on EKF training algorithm has a good function approximate performance.

Keywords--Intuitionistic fuzzy neural networks;training algorithm;Extended Kalman Filter

\section{INTRODUCTION}

Intuitionistic fuzzy sets(IFSs) are introduced by Atanassov[1][2], which are generalization of the concept of fuzzy sets by adding an additional attribute parameter called non-membership[3]. IFSs are successful applied in many areas, multi-criteria fuzzy decision-making[4][5], pattern recognition[6], air quality modelling[7], time series prediction[8], etc.

During the past decade, a fuzzy neural network has been found to solve many problems which cannot be solved before[9]. For instance, the fuzzy neural network has been successfully applied in system identification[10], intelligent control[11], etc. Since IFSs have proved to be more powerful to deal with vagueness and uncertainty than fuzzy sets, combination of IFSs and artificial neural networks is investigated by many experts. In [12], a max-min intuitionistic fuzzy Hopfield neural network (IFHNN) is proposed. In [13], an intuitionistic fuzzy neural model is present based on an simple intuitionistic inference system. In [14], an intuitionistic fuzzy inference system based on game theory is proposed. In[15], an intuitionsitic fuzzy neural network based on two steps gradient descent algorithm is present. In this paper, an intuitionistic fuzzy neural network based on EKF training algorithm is present. The EKF procedure to update intuitionistic fuzzy parameters is introduced. An example is given to show the EKF training yield more improved performance than using gradient descent algorithm.

This paper is organized as follows. In Section II, the new intuitionistic fuzzy inference system will be introduced. In Section III, a intuitionistic fuzzy neural network model with five layers is builded. In Section IV, the EKF training algorithm will be given. In Section V, An example is given to show the EKF training yield more

\author{
Lili Zhang
}

Institute College of Opto-electronic Engineering

Changchun University of Science and Technology

ChangChun 130022, China

zhangliligf@163.com

improved performance than using gradient descent algorithm. The conclusion and topics for future research are drawn in section VI.

\section{INTUITIONISTIC FUZZY INFERENCE SYSTEM}

\section{A. Intuitionistic fuzzy set}

In [1]Atanassov defines an intuitionistic fuzzy set(IFS) $A$ over a finite universal set $E$ as an object having the following form:

$$
A=\left\{\left(x, \mu_{A}(x), \gamma_{A}(x)\right) \mid x \in X\right\}
$$

Where $, \mu_{A}: X \rightarrow[0,1]$ and $\gamma_{A}: X \rightarrow[0,1]:$ are such that $0 \leq \mu_{A}+\gamma_{A} \leq 1, \mu_{A}(x)$ denote a degree of membership of $x \in A, \gamma_{A}(x)$ denote a degree of non-membership of $x \in A$. For each intuitionistic fuzzy set in $X$, we call $\pi_{A}(x)=1-\mu_{A}(x)-\gamma_{A}(x)$ a "hesitation margin" of $x \in A$, it expresses a hesitation degree of whether $x$ belongs to $A$ or not. It is obvious that $0 \leq \pi_{A} \leq 1$, for each $x \in X$.

\section{B. Intuitionistic fuzzy inference system(IFIS)}

A new IFIS is defined in [14]. Then it is possible to define its output $y_{\eta}$ as

$$
y_{\eta}=\left(-\pi_{A}(x) y_{i}+\pi_{A}(x y)\right.
$$

Where $y_{\eta}$ is output of the FIS using the membership function $\mu_{A}(x), y_{\gamma}$ is the output of the FIS using the non-membership function $\gamma_{A}(x)$. For $\pi=0$, output $y_{\eta}$ will reduce to the output of a traditional fuzzy system.

\section{INTUITIONISTIC FUZZY NEURAL NETWORKS}

An intuitionistic fuzzy neural network system with five layers structure is shown in fig1. The first layer is input layer. The second layer is membership and non-membership function creating layer. The third layer is inference layer. The fourth layer is normalized layer. The fifths layer is output layer.

Layer 1: no computation is done in this layer, it just pass inputs in to next layer.

Layer 2: the membership and non-membership are determined for the neurons generation criteria: 


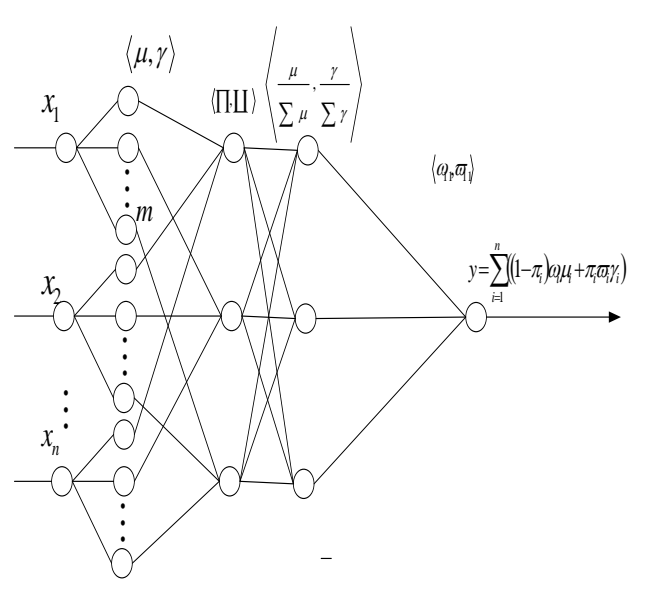

Figure 1. IFNN architecture

$$
\begin{gathered}
\mu(x)=\exp \left(\frac{-(x-c)^{2}}{2 \sigma^{2}}\right) \\
\gamma(x)=\left(1-\exp \left(\frac{-(x-c)^{2}}{2 \sigma^{2}}\right)\right)^{k}, k \geq 1
\end{gathered}
$$

Where $c 、 \sigma 、 k$ are coefficient, which need to be designed

Layer 3:It is fuzzy inference layer. Each node represents a fuzzy rule. The degree of fulfillment and non-fulfillment of the $i$ th rule is represented by the following equations:

$$
\begin{aligned}
& \bar{\mu}_{j}=\mu_{1 j} \mu_{2 j} \cdots \mu_{n j}=\prod_{i=1}^{n} \mu_{i j} \\
& \bar{\gamma}_{j}=\gamma_{1 j} \gamma_{2 j} \cdots \gamma_{n j}=\prod_{i=1}^{n} \gamma_{i j}
\end{aligned}
$$

Layer 4: It normalized the degree of fulfillment and non-fulfillment of the fuzzy and calculated the hesitation margin index.

$$
\begin{gathered}
\bar{\varphi}_{j}=\frac{\bar{\mu}_{j}}{\sum_{j=1}^{m} \bar{\mu}_{j}} \\
\bar{\phi}_{j}=\frac{\bar{\gamma}_{j}}{\sum_{j=1}^{m} \bar{\gamma}_{j}} \\
\pi_{j}=1-\bar{\varphi}_{j}-\bar{\phi}_{j}
\end{gathered}
$$

Layer 5: The output of the intuitionistic neural network with $n$ rules can be calculated as:

$$
y=\sum_{j=1}^{m}\left(\left(1-\pi_{j}\right) \omega_{j} \bar{\varphi}_{j}+\pi_{j} \varpi_{j} \bar{\phi}_{j}\right)=\sum_{j=1}^{m} y_{j}
$$

\section{IFNN TRAINING ALGORITHM BASED ON EXTENDED KALMAN FILTER}

\section{A. An training algoritm based on EKF}

The IFNN architecture consists of two trainable parameter sets:
(1):The polynomial parameters $\langle\omega, \varpi\rangle$.

(2):The membership and non-membership function parameter $[c, \sigma, k]$.

The polynomial parameters $\langle\omega, \varpi\rangle$ can be solved by least square regression techniques[15].

So in this part, only a training algorithm based on extended kalman filter how to optimize the membership and non-membership function parameter is introduced.

A intuitinoistic fuzzy neural network's behaviour can be described by the following nonlinear discrete-time system[16]:

$$
\begin{gathered}
w_{k+1}=w_{k}+\omega_{k} \\
y_{k}=h_{k}\left(w_{k}, u_{k}, v_{k-1}\right)+v_{k}
\end{gathered}
$$

The first equation, known as the process equation, where the state of the system is given by the network's membership and non-membership function parameter $w_{k}=\left[c_{k}, \sigma_{k}, k_{k}\right]$.The process noise $\omega_{k}$ is characterized as aero-mean, white noise with covariance given by $E\left[\omega_{k} \omega_{l}^{T}\right]=\delta_{k, l} Q_{k}$.The second equation, known as the observation or measurement equation, represents the network's desired response vector $y_{k}$ as a nonlinear function of the input vector $u_{k}$, the network's membership and non-membership function parameter $w_{k}$, and the recurrent node activations $v_{k}$. The measurement noise $v_{k}$ is typically characterized as aero-mean, white noise with covariance given by $E\left[v_{k} v_{l}^{T}\right]=\delta_{k, l} R_{k}$

The training problem using Extended Kalman filter theory can now be described as finding the minimum mean-squared error estimate of the state $w$ using all observed data so far. The Extended Kalman filter solution to the training problem is given by the following recursion[17]:

$$
\begin{gathered}
A_{k}=\left[R_{k}+H_{k}^{T} P_{k} H_{k}\right]^{-1} \\
K_{k}=P_{k} H_{k} A_{k} \\
\hat{w}_{k+1}=\hat{w}_{k}+K_{k} \xi_{k} \\
P_{k+1}=P_{k}-K_{k} H_{k}^{T} P_{k}+Q_{k}
\end{gathered}
$$

Where $k$ is the discrete time index; the vector $\hat{w}_{k}$ is the estimate of the state of the system at update step $k ; K_{k}$ is the Kalman gain matrix; $\xi_{k}=y_{k}-\hat{y}_{k}$ is error vector, where $y_{k}$ is the target vector and $\hat{y}_{k}$ is the network's output vector; $P_{k}$ is error covariance matrix; $R_{k}$ and $Q_{k}$ is measurement and process noise covariance matrices.

The extended Kalman filter training is carried out in a sequential fashion, one step of training involves the following steps: 


\section{Initialize:}

The number of iteration, $n$

$c_{i}=\left[c_{i 1}, c_{i 2}, \cdots, c_{i m}\right]$ in the range of $\left[c_{\min }, c_{\max }\right]$

$\sigma_{i}=\left[\sigma_{i 1}, \sigma_{i 2}, \cdots, \sigma_{i m}\right]$ in the range of

$\left[\sigma_{\min }, \sigma_{\max }\right]$

$k_{i}=\left[k_{i 1}, k_{i 2}, \cdots, k_{i m}\right]$ in the range of $\left[k_{\text {min }}, k_{\text {max }}\right]$

$P_{c_{i}}(0), P_{\sigma_{i}}(0), P_{k_{i}}(0)$ each equals to an identify matrix of $m \times m$

Recursion:

For $k=1,2, \cdots, n$ do

1. Calculate the membership $\mu_{i j}(x)$ and non-membership $\gamma_{i j}(x)$;

2. Calculate the Jacobians $H_{c_{i}}(k) H_{\sigma_{i}}(k)$ and $H_{k_{i}}(k)$ respectively.

3. Calculate the Kalman gain matrices $K_{c_{i}}(k)$

$K_{\sigma_{i}}(k)$ and $K_{k_{i}}(k)$;

4. Update the error covariance matrix $P_{c_{i}}(k)$, $P_{\sigma_{i}}(k)$ and $P_{k_{i}}(k)$;

5. Update the parameter $c_{i} \sigma_{i}$ and $k_{i}$.

B. Derivation of Extended Kalman Filter based on adaptation algoritm

The Jacobians $H_{c_{i}}(k) H_{\sigma_{i}}(k)$ and $H_{k_{i}}(k)$ are calculated as followed:

$H_{c_{i}}(k)=$

$\frac{\partial y}{\partial y_{j}}\left(\begin{array}{l}\frac{\partial y_{j}}{\partial \pi_{j}}\left(\frac{\partial \pi_{j}}{\partial \bar{\varphi}_{j}} \frac{\partial \bar{\varphi}_{j}}{\partial \bar{\mu}_{j}} \frac{\partial \bar{\mu}_{j}}{\partial \mu_{i j}} \frac{\partial \mu_{i j}}{\partial c_{i j}}+\frac{\partial \pi_{j}}{\partial \bar{\phi}_{j}} \frac{\partial \bar{\phi}_{j}}{\partial \bar{\gamma}_{j}} \frac{\partial \bar{\gamma}_{j}}{\partial \gamma_{i j}} \frac{\partial \gamma_{i j}}{\partial c_{i j}}\right)+ \\ \frac{\partial \bar{\varphi}_{j}}{\partial \bar{\varphi}_{j}} \frac{\partial \bar{\mu}_{j}}{\partial \mu_{i j}} \frac{\partial \mu_{i j}}{\partial c_{i j}}+\frac{\partial y_{j}}{\partial \bar{\phi}_{j}} \frac{\partial \bar{\phi}_{j}}{\partial \bar{\gamma}_{j}} \frac{\partial \bar{\gamma}_{j}}{\partial \gamma_{i j}} \frac{\partial \gamma_{i j}}{\partial c_{i j}}\end{array}\right)$

Because $y=\sum_{j=1}^{m} y_{j}$ so $\frac{\partial y}{\partial y_{j}}=1$

$\gamma(x)=\left(1-\exp \left(\frac{-\left(x-c_{i j}\right)^{2}}{2 \sigma_{i j}^{2}}\right)\right)^{k_{i j}}, k_{i j} \geq 1$ so

$\frac{\partial \gamma_{i j}}{\partial k_{i j}}=k_{i j}\left(1-\exp \left(\frac{-\left(x-c_{i j}\right)^{2}}{2 \sigma_{i j}{ }^{2}}\right)\right)^{k_{i j}-1}=k_{i j} \frac{\gamma_{i j}}{1-\mu_{i j}}$

Substituting these into the update equation we get
$H_{c_{i}}(k)$

$=\left(-\omega_{j} \bar{\varphi}_{j}+\varpi_{j} \bar{\phi}_{j}\right)\left(\begin{array}{c}-\left(\frac{1-\bar{\varphi}_{j}}{\sum_{j=1}^{m} \bar{\mu}_{j}}\right) \frac{\bar{\mu}_{j}}{\mu_{i j}} \mu_{i j} \frac{\left(x-c_{i j}\right)}{\sigma_{i j}{ }^{2}}+ \\ \left(\frac{1-\bar{\phi}_{j}}{\sum_{j=1}^{m} \bar{\gamma}_{j}}\right) \frac{\bar{\gamma}_{j}}{\gamma_{i j}} k \frac{\gamma_{i j}}{1-\mu_{i j}} \mu_{i j} \frac{\left(x-c_{i j}\right)}{\sigma_{i j}{ }^{2}}\end{array}\right)+$

$\left(1-\pi_{j}\right) \omega_{j}\left(-\left(\frac{1-\bar{\varphi}_{j}}{\sum_{j=1}^{m} \bar{\mu}_{j}}\right) \frac{\bar{\mu}_{j}}{\mu_{i j}} \mu_{i j} \frac{\left(x-c_{i j}\right)}{\sigma_{i j}^{2}}\right)+$

$\pi_{j} \varpi_{j}\left(\frac{1-\bar{\phi}_{j}}{\sum_{j=1}^{m} \bar{\gamma}_{j}}\right) \frac{\bar{\gamma}_{j}}{\gamma_{i j}} k \frac{\gamma_{i j}}{1-\mu_{i j}} \mu_{i j} \frac{\left(x-c_{i j}\right)}{\sigma_{i j}^{2}}$

$H_{\sigma_{i}}(k)$

$=\left(-\omega_{j} \bar{\varphi}_{j}+\varpi_{j} \bar{\phi}_{j}\right)\left(\begin{array}{l}\left(\frac{1-\bar{\varphi}_{j}}{\sum_{j=1}^{m} \bar{\mu}_{j}}\right) \frac{\bar{\mu}_{j}\left(x-c_{i j}\right)^{2}}{\sigma_{i j}{ }^{3}}- \\ \left(\frac{1-\bar{\phi}_{j}}{\sum_{j=1}^{m} \bar{\gamma}_{j}}\right) \frac{k_{i j} \bar{\gamma}_{j}}{1-\mu_{i j}} \mu_{i j} \frac{\left(x-c_{i j}\right)^{2}}{\sigma_{i j}{ }^{3}}\end{array}\right)+$

$\left(1-\pi_{j}\right) \omega_{j}\left(\left(\frac{1-\bar{\varphi}_{j}}{\sum_{j=1}^{m} \bar{\mu}_{j}}\right) \frac{\bar{\mu}_{j}\left(x-c_{i j}\right)^{2}}{\sigma_{i j}{ }^{3}}\right)-$

$\pi_{j} \varpi_{j}\left(\frac{1-\bar{\phi}_{j}}{\sum_{j=1}^{m} \bar{\gamma}_{j}}\right) \frac{k_{i j} \bar{\gamma}_{j}}{1-\mu_{i j}} \mu_{i j} \frac{\left(x-c_{i j}\right)^{2}}{\sigma_{i j}^{3}}$

$H_{k_{i}}(k)=\left(-\omega_{j} \bar{\varphi}_{j}+\varpi_{j} \bar{\phi}_{j}\right)\left(\frac{1-\bar{\phi}_{j}}{\sum_{j=1}^{m} \bar{\gamma}_{j}}\right) \frac{\bar{\gamma}_{j}}{\gamma_{i j}} k \frac{\gamma_{i j}}{1-\mu_{i j}}+$

$\pi_{j} \varpi_{j}\left(\frac{1-\bar{\phi}_{j}}{\sum_{j=1}^{m} \bar{\gamma}_{j}}\right) \frac{\bar{\gamma}_{j}}{\gamma_{i j}} k \frac{\gamma_{i j}}{1-\mu_{i j}}$

$=\left(-\omega_{j} \bar{\varphi}_{j}+\varpi_{j} \bar{\phi}_{j}+\pi_{j} \varpi_{j}\left(\frac{1-\bar{\phi}_{j}}{\sum_{j=1}^{m} \bar{\gamma}_{j}}\right) \frac{k_{i j} \bar{\gamma}_{j}}{1-\mu_{i j}}\right.$

\section{EXAMPLE}

In this section, the effectiveness of the intuitionistic fuzzy neural based on EKF training algorithm is demonstrated. The simulation is carried out in the unified running environment of matlab2011a. 
In the experiment, the function $\sin (\mathrm{x})$ is needed to be approximated:

$$
f(x)=\sin (x)
$$

The intuitionistic fuzzy neural network is constructed with four fuzzy rules. A random sampling of the interval $[-4,4]$ is used in obtaining 200input-output data pairs for the training set. $c_{i}$ in the range of $[-5,5], \sigma_{i}$ in the range of $[1,4], k_{i}$ in the range of $[1,5], \omega_{i}, \varpi_{i}$ in the range of $[-1,1]$.Fig 2 shows the training results and training results. We can see that the resulting intuitionistic fuzzy neural network can approximate well to the original function. Comparisons of the gradient descent algorithm, Fig 4 plot root mean squared error during the training process of EKF and gradient descent algorithm. The results illustrate the good approximate performance.
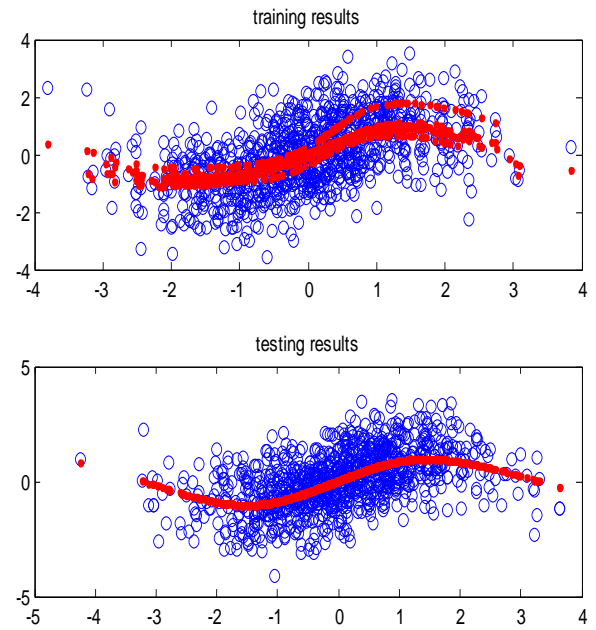

Figure 2. training and testing results

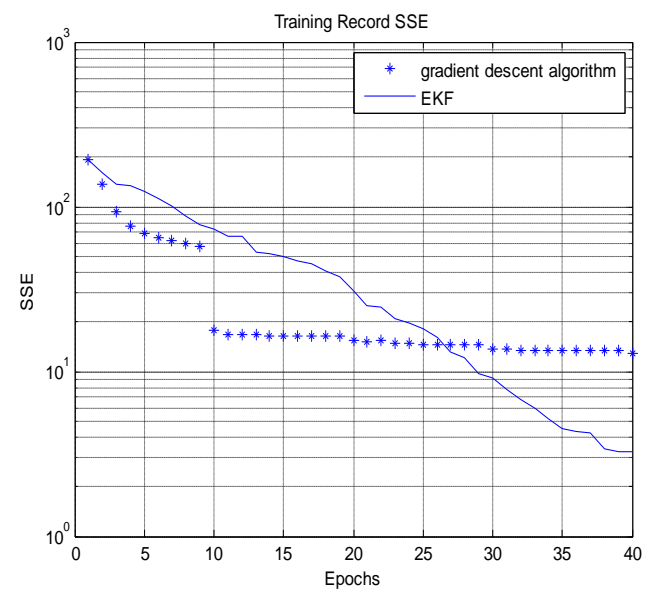

Figure 3. SSE training performance

\section{CONCLUSION}

In this paper, a training algorithm based on EKF for intuitionistic fuzzy neural has been developed. An intuitionistic fuzzy neural network with five layers has been constructed. The EKF has been applied to parameter identification of intuitionistic fuzzy neural network. The EKF procedure to update parameter is introduced. The derivation of EKF based on adaptation algorithm for intuitionistic fuzzy adaptive equalizer is given. Simulation results show that the intuitionistic fuzzy neural network based on EKF training algorithm has a good function approximate performance than gradient descent training algorithm.

\section{REFERENCES}

[1] K. Atanassov, "Intuitionistic fuzzy sets“, Fuzzy Sets and Systems, vol .20, Nov 1986, pp. 87-96.

[2] K. Atanassov, "More on Intuitionistic fuzzy sets", Fuzzy Sets and Systems, vol 33,Mar 1989, pp. 37-46.

[3] K. Atanassov, "Intuitionistic Fuzzy Sets: Theory and Applications", Physica Verlag, Wyrzburg, 1999.

[4] GuiwuWei, WendeYi, "Method for Intuitionistic fuzzy multiple attribute decision making without weight information but with preference information on alternatives",2008 Chinese Control and Decision Conference, 1974-1974,2008

[5] A.S.Xu, "Multi-person multi-attribute decision making models under intuitionistic fuzzy environment", Fuzzy Optimal and Decision Making,vol 6, Dec, 2007 :221-236

[6] D.F. Li, C.T. Cheng, "New similarity measures of intuitionistic fuzzy sets and application to pattern recognitions", Pattern Recognition Letters, vol 23 ,Oct 2002,pp.221-225.

[7] V. Olej, P. Hajek," Air Quality Classification by Intuitionistic Fuzzy Relations and Kohonen' s Selforganizing Feature Maps”, Scientific Papers Univerzity of Pardubice, Faculty of Economics and Administration, vol 8, Dec, 2008, pp.146-152.

[8] V. Olej, "IF-Inference Systems Design for Prediction of Ozone Time Series", Proceedings of the 20th International Conference on Artificial Neural Networks, Thessaloniki, Greece, 2010.

[9] Liang Y.C., Feng D.P., Liu G.R., Yang X.W. and Han X., "Neural identification of rock parameters using fuzzy adaptive learning parameters", Computers \& Structures,vol 81,Jan 2003, pp. 2373-2382,

[10] I.S. Baruch, Q.B. Lopez and J.M. Flores," A fuzzy-neural multi-model for nonlinear systems identification and control", Fuzzy Sets and Systems, vol 159,Jan 2008, pp. 2650-2667.

[11] C.F. Hsua, P.Z. Linb, T.T. Lee and C.H. Wang, "Adaptive asymmetric fuzzy, Fuzzy Sets and Systems", Vol.79, Apr 1996,pp.403-405.

[12] Long Li, Jie Yang, Wei Wu, "Intuitionistic fuzzy Hopfield neural network and its stability", Expert Systems with Applications ,vol 29 ,Jan 2005, pp. 589-597.

[13] Lei Ying-jie, Lu Yan-li, Li Zhao-yuan, "Function approximation capabilities of intuitionistic fuzzy reasoning neural networks", Control and Decision, vol 5, April 2007, pp. 596-600 (in chinese).

[14] Li Songwei, ZhouXiaoguang, "Takagi-Sugeno intuitionistic fuzzy inference systems based on game theory", The 2th international conference on computer science and service system(CSSS 2012), vol5, Aug 2012, pp3392-3396

[15] Zhou Xiaoguang, ZhaoRenhou,"An intuitionistic fuzzy neural network with triangular membership function",Lecture notes in elcetrical engineering in presss

[16] Wai Kit Wong, Heng siong Lim,"Extended Kalman Filter based on fuzzy adaptive filer",Klaman Filter:Recent Adavances and Applications, ISBN 978-953-307-000-1,pp.584,April 2009,I-Tech, Vienna,Austria

[17] Simon Haykin,"Kalman Filtering and Neural Network ", 2001 , John Wiley\&Sons, Inc A WILEY-INTERSCIENCE PUBLICATION, NEW YORK 